\title{
Non-birational Calabi-Yau threefolds that are derived equivalent
}

\author{
Andrei Căldăraru*
}

\begin{abstract}
We argue that the existence of genus one fibrations with multisections of high degree on certain Calabi-Yau threefolds implies the existence of pairs of such varieties that are not birational, but are derived equivalent. It also (likely) implies the existence of nonbirational counterexamples to the Torelli problem for Calabi-Yau threefolds.
\end{abstract}

\section{Introduction}

0.1. The purpose of this paper is to prove the following theorem.

Theorem. Assume that there exists a smooth, projective Calabi-Yau threefold $\mathrm{X}$ with the following properties:

1. The Picard number of $\mathrm{X}$ is 2 ,

$$
\rho=\operatorname{rk} \operatorname{Pic}(X)=2 \text {; }
$$

2. $\mathrm{X}$ admits a non-isotrivial genus one fibration $\mathrm{X} \rightarrow \mathrm{S}$ of fiber degree $\mathrm{n}$;

3. the group $\mathrm{U}\left(\mathbf{Z}_{n}\right) / \mathbf{Z}_{2}$ has at least 3 elements $\left(\mathbf{Z}_{2}\right.$ acts by negation on $\left.\mathbf{Z}_{n}\right)$;

4. $\mathrm{X} \rightarrow \mathrm{S}$ has at worst isolated non-reduced fibers.

Then there exist smooth, projective Calabi-Yau threefolds $\mathrm{X}^{\prime}$ and $\mathrm{X}^{\prime \prime}$ that are not birational and have equivalent derived categories, $\mathbf{D}\left(\mathrm{X}^{\prime}\right) \cong \mathbf{D}\left(\mathrm{X}^{\prime \prime}\right)$. Furthermore, $\mathrm{X}^{\prime}$ and $\mathrm{X}^{\prime \prime}$ have isomorphic Z[1/2]-Hodge structures.

0.2. The interest in a theorem of the type described above is threefold (pun intended). In the first place, we are interested in understanding the classification problem of FourierMukai equivalence classes of varieties (varieties that have equivalent derived categories). This topic has been a central one in recent years in algebraic geometry, having implications to the study of moduli spaces of sheaves, the Mori program, and mirror symmetry. In dimensions 1 and 2 the classification is complete by work of Mukai [15], [16], BondalOrlov [1, Orlov [17, and Bridgeland-Maciocia [4. It is also known that the problem is

*Mathematics Department, University of Wisconsin-Madison, 480 Lincoln Drive, Madison, WI 537061388, USA, e-mail: andreic@math.wisc.edu 
essentially only interesting for Calabi-Yau varieties. This follows from results of BondalOrlov [1] and Toda 20. In dimension 3, we know that birational Calabi-Yau threefolds are Fourier-Mukai equivalent by work of Bridgeland [3], but no examples of non-birational Calabi-Yau threefolds with equivalent derived categories are known.

0.3. Question. Is there a pair of Fourier-Mukai equivalent Calabi-Yau threefolds that are not birational?

0.4. Theorem 0.1 should be regarded as strong evidence that the answer to Question 0.3 is "yes." Indeed, plenty of examples of varieties satisfying the conditions of Theorem 0.1 but with $p \leq 6$ are known, and there is no apparent reason why $p \leq 6$ would be a limitation. The only reason we can not find explicit examples of varieties with the required properties is the fact that we do not know explicit equations for elliptic curves of degree 7 or more.

0.5. Certain aspects of string theory also provide motivation for trying to understand Fourier-Mukai equivalent varieties. Kontsevich's Homological Mirror Symmetry conjecture [14] predicts an equivalence of triangulated categories between the derived category of $X$ and the Fukaya category of $\check{X}$, when $X$ and $\check{X}$ are a mirror pair. If $X^{\prime}$ and $X^{\prime \prime}$ have the same derived category, it is expected that they should have the same mirror as well. Such examples are very interesting to study.

More generally, the derived category of $\mathrm{X}$ is conjectured to encode the entire B-model topological quantum field theory of the open string theory compactified on $X[13$. Furthermore, it has been argued [12] that this data should also be sufficient for determining the closed string topological quantum field theory as well. Thus Fourier-Mukai equivalent Calabi-Yau threefolds must yield equivalent B-model TQFT's, and thus it is useful to have examples at hand.

0.6. In a third direction, we are interested in understanding how the Torelli principle fails for Calabi-Yau threefolds. The Torelli problem asks how much information about a space $X$ can be recovered from Hodge data on $X$. For example, a typical Torelli-type theorem asserts that if two K3 surfaces have isomorphic Z-Hodge structures, then they are themselves isomorphic. If two spaces of the same type (say, Calabi-Yau threefolds) have isomorphic Hodge structures but are not themselves isomorphic, we say that they fail the Torelli principle.

For Calabi-Yau threefolds this is known to happen. Szendröi's examples [19] are pairs of Calabi-Yau threefolds that are deformation equivalent, have isomorphic Z-Hodge structures, but are not isomorphic. However, the varieties in these examples are pairwise birational, so one may ask if all failures of Torelli for Calabi-Yau threefolds are limited to birational varieties.

Theorem 0.1 should be viewed as evidence that Torelli might fail even for non-birational examples. While we are unable to prove a statement about Z-Hodge structures, we argue that the varieties $X^{\prime}$ and $X^{\prime \prime}$ constructed in the theorem have isomorphic $Z$ [1/2]-Hodge structures, and we give a convincing argument that the isomorphism of Hodge structures might actually extend to one over $\mathbf{Z}$. Thus $X^{\prime}$ and $X^{\prime \prime}$ are very close to providing an example of a non-birational failure of Torelli. 
0.7. The paper is organized as follows. We collect in Section 1 required results about Fourier-Mukai transforms and genus one fibrations. We also include an easy example of a variety satisfying the conditions of Theorem 0.1 with $n=3$. In Section 2 we prove the existence of Fourier-Mukai partners that are not birational. An amusing aspect of the construction is the fact that the proof of non-birationality is not constructive. Explicitly, we construct three varieties that are Fourier-Mukai partners, and we argue that at most two of them can be birational. We conclude with Section 3 where we prove that $X^{\prime}$ and $X^{\prime \prime}$ of Theorem 0.1 have isomorphic Z[1/2]-Hodge structures, and we discuss what is needed to obtain an isomorphism over $\mathbf{Z}$.

0.8. Acknowledgments. This paper grew out of conversations with Mark Gross, Paul Aspinwall, and Igor Dolgachev. Input on the Torelli problem was provided by Balazs Szendrői.

\section{Preliminaries}

In this section we collect several results about Fourier-Mukai transforms and genus one fibrations.

1.1. Let $X$ and $Y$ be smooth, projective varieties. Given an object $E$ of $D(X \times Y)$, define the integral transform $\Phi_{\mathrm{X} \rightarrow \mathrm{Y}}^{\mathrm{E}}$ with kernel $\mathrm{E}$ to be the functor $\mathrm{D}(\mathrm{X}) \rightarrow \mathbf{D}(\mathrm{Y})$ given by

$$
\Phi_{\mathrm{X} \rightarrow \mathrm{Y}}^{\mathrm{E}}(-)=\mathbf{R} \pi_{\mathrm{Y}, *}\left(\pi_{\mathrm{X}}^{*}(-) \stackrel{\mathrm{L}}{\otimes} \mathrm{E}\right),
$$

where $\pi_{X}, \pi_{Y}$ are the projections from $X \times Y$ to $X$ and $Y$, respectively.

If an integral transform is an equivalence, it is called a Fourier-Mukai transform. It is known [17] that all equivalences arise as integral transforms.

1.2. Following Mukai, we associate to any integral transform $\Phi=\Phi_{X \rightarrow Y}^{\mathrm{E}}$ a map on cohomology

$$
\varphi=\varphi_{\mathrm{X} \rightarrow \mathrm{Y}}^{\mathrm{E}}: \mathrm{H}^{*}(\mathrm{X}, \mathrm{Q}) \rightarrow \mathrm{H}^{*}(\mathrm{Y}, \mathrm{Q})
$$

by the formula

$$
\varphi_{\mathrm{X} \rightarrow \mathrm{Y}}^{\mathrm{E}}(-)=\pi_{Y, *}\left(\pi_{X}^{*}(-) \cdot v(\mathrm{E})\right) .
$$

Here $v(\mathrm{E}) \in \mathrm{H}^{*}(\mathrm{X} \times \mathrm{Y}, \mathbf{Q})$ is the Mukai vector of $\mathrm{E}$,

$$
v(E)=\operatorname{ch}(E) \cdot \sqrt{t_{d}} \text {. }
$$

This association is functorial, in the sense that the map associated to the identity functor is the identity on $\mathrm{H}^{*}(X, \mathbf{Q})$, and the map associated to $\Psi \circ \Phi$ is $\psi \circ \varphi$, if $\psi$ is associated to $\Psi$ and $\varphi$ to $\Phi$.

1.3. Extending this construction to complex cohomology, the map $\varphi$ associated to an integral transform preserves the Hochschild grading. Explicitly, for every integer $i, \varphi$ restricts to a map

$$
\varphi: \bigoplus_{\mathrm{q}-\mathrm{p}=\mathrm{i}} \mathrm{H}^{\mathrm{p}, \mathrm{q}}(\mathrm{X}) \rightarrow \bigoplus_{\mathrm{q}-\mathrm{p}=\mathrm{i}} \mathrm{H}^{\mathrm{p}, \mathrm{q}}(\mathrm{Y})
$$


1.4. We have argued in [7] that Mukai's original pairing from [16] on the cohomology of K3 surfaces can be generalized to arbitrary varieties. For simplicity we restrict our attention to Calabi-Yau varieties only, and then the generalized Mukai pairing on $\mathrm{H}^{*}(\mathrm{X}, \mathbf{Q})$ is the map

$$
\langle-,-\rangle: H^{*}(X, Q) \otimes H^{*}(X, Q) \rightarrow \mathbf{C}
$$

defined by

$$
\langle v, w\rangle=\int_{X} v^{\vee} \cdot w
$$

where, for a vector $v=\sum v_{\mathrm{k}}$ with $v_{\mathrm{k}} \in \mathrm{H}^{\mathrm{k}}(\mathrm{X}, \mathrm{Q})$,

$$
v^{\vee}=\sum i^{k} v_{k}
$$

(Here $i=\sqrt{1}$.) Adjoint functors give rise to adjoint maps on cohomology with respect to the Mukai pairing, and therefore if $\Phi: \mathrm{D}(\mathrm{X}) \rightarrow \mathrm{D}(\mathrm{Y})$ is an equivalence, then $\varphi$ is an isometry between $\mathrm{H}^{*}(\mathrm{X}, \mathbf{Q})$ and $\mathrm{H}^{*}(\mathrm{Y}, \mathbf{Q})$, endowed with the Mukai pairings.

1.5. Let $X$ be a smooth, connected projective variety. A flat projective morphism $X \rightarrow S$ to a smooth variety $S$ is called a genus one fibration if its generic fiber $X_{\eta} \rightarrow \eta$ is a smooth curve of genus one over the function field $\eta$ of $S$. In general $X_{\eta}$ is not expected to have any closed points, as $\eta$ is not algebraically closed. Equivalently, the map $X \rightarrow S$ is not expected to have any rational sections. However, there is always a finite field extension $\eta^{\prime} \rightarrow \eta$ over which $X_{\eta^{\prime}}$ does have a section. The smallest degree of such an extension is called the fiber degree of the fibration $X \rightarrow S$. It is also the smallest positive degree of the restriction of a relatively ample divisor on $X / S$ to a smooth fiber of $X \rightarrow S$.

Fix a relatively ample polarization of $X / S$, which will be implicit from now on. Consider the relative moduli space (in the sense of Simpson [18]) of stable sheaves on the fibers of $\mathrm{X} / \mathrm{S}$ having the same Hilbert polynomial as a line bundle of degree $\mathrm{k}$ on a smooth fiber of $X / S$. It has a unique component containing a point corresponding to a line bundle on a smooth fiber of $X / S$. We'll denote this component by $X^{(k)}$. From the construction, it comes with a natural quasi-projective morphism to $S$. If $s \in S$ is a closed point such that $X_{s}$ is smooth, then the fiber of $X^{(k)}$ over $s$ is isomorphic to the moduli space of line bundles of degree $k$ on $X_{s}$. Thus $X_{s}^{(k)}$ is isomorphic to $X_{s}$, although not canonically (the choice of such an isomorphism depends on the choice of a point on $X_{s}$, and such a choice can not be made globally on $X$ unless $X \rightarrow S$ admits a section).

Now assume that $X$ has dimension at most 3. It was argued by Bridgeland and Maciocia [4] that if $k$ is coprime to the degree of the polarization on a smooth fiber, then every semistable torsion free sheaf on a fiber of $X / S$ is stable, and therefore $X / S$ is projective. Furthermore, $X^{(k)}$ is smooth, a universal sheaf $E_{0}$ for the moduli problem considered exists on $X \times{ }_{S} X^{(k)}$, and extending $E_{0}$ by zero to $X \times X^{(k)}$ gives rise to a kernel $E$ which induces a Fourier-Mukai transform

$$
\Phi_{X \rightarrow X^{(k)}}^{\mathrm{E}}: \mathbf{D}(X) \stackrel{\sim}{\longrightarrow} \mathbf{D}\left(X^{(k)}\right) .
$$

Thus $X^{(k)} \rightarrow S$ is a genus one fibration, Fourier-Mukai equivalent to $X$. 
1.6. For the sake of completeness, we include an example from [6]. Let $X$ be a general hypersurface of bidegree $(3,3)$ in $\mathbf{P}^{2} \times \mathbf{P}^{2}$. It is a smooth Calabi-Yau threefold of Picard number 2. Projection from $X$ to any one of the two $\mathbf{P}^{2}$ 's gives $X$ the structure of a genus one fibration of fiber degree 3. A similar example with fiber degree 5 can be found in [loc. cit.].

\section{A proof of non-birationality}

In this section we prove, assuming given a space $X$ with the properties required by Theorem 0.1, that there exists a pair of Calabi-Yau threefolds $X^{\prime}$ and $X^{\prime \prime}$ which are derived equivalent but not birational.

2.1. The idea of the construction is to consider the various powers $X^{(k)}$ of $X$, for $k$ coprime to $n$, the fiber degree of $X / S$. They are all Calabi-Yau threefolds, admitting a genus one fibration structure, and they are all Fourier-Mukai equivalent to each other. In the general case, we'll argue that there are at least three non-isomorphic such powers of $X$. On the other hand, we will see that in the birational equivalence class of $X=X^{(1)}$ there is at most one other space that admits a genus one fibration. Therefore we conclude that at least two of the spaces $X^{(\mathrm{k})}$ are not birational to each other.

There is one special case to consider, namely when all the $X^{(\mathrm{k})}$ spaces, admit not just one, but two genus one fibrations. In this case we'll argue that each one of the $X^{(k)}$ spaces is unique in its birational equivalence class, but at least two of these spaces are non-isomorphic. Thus, again, we are able to find non-birational spaces which are Fourier-Mukai equivalent.

2.2. Proposition. For $k$ and $k^{\prime}$ coprime to $n, X^{(k)}$ is isomorphic to $X^{\left(k^{\prime}\right)}$ as fibrations over $S$ (with induced structure from $X / S$ ) if and only if $k^{\prime}= \pm k \bmod n$.

Proof. Assume that $X^{(\mathrm{k})}{ }_{\text {iso }} X^{\left(\mathrm{k}^{\prime}\right)}$ as fibrations over $\mathrm{S}$. The idea of the proof is to argue that there exists an automorphism of $\mathrm{J} / \mathrm{S}$, the relative Jacobian of $\mathrm{X} / \mathrm{S}$, that maps the Brauer class representing $X^{(k)}$ to the Brauer class of $X^{\left(k^{\prime}\right)}$. We then argue that the only such automorphism possible is negation along the fibers of $\mathrm{J} / \mathrm{S}$, which implies that $\mathrm{k}^{\prime}=$ $\pm k \bmod n$.

Let $\mathrm{J} / \mathrm{S}=\mathrm{X}^{(0)} / \mathrm{S}$ be the relative Jacobian of $\mathrm{X} / \mathrm{S}$. We will only be interested in properties of the generic fiber of $J / S$, so we will not be concerned with compactifying J. Let $\eta$ be the generic point of $S$, and consider $J_{\eta}$, the generic fiber of $J / S$. It is a smooth elliptic curve over the non-algebraically closed field $\eta$.

As argued in [6], there is a distinguished cohomology class $\alpha$ in $\operatorname{Br}\left(J_{\eta} / \eta\right)$, the Brauer group of $\mathrm{J}_{\eta}$, corresponding to the original fibration $X / S$. The class $\alpha$ can be constructed either by means of Ogg-Shafarevich theory, or as an obstruction class to the existence of a universal sheaf on $X \times_{\eta}$ J. See [5] or [6] for details.

By [6, 6.5], we can find an identification of the relative Jacobian of $X^{(k)} / S$ with J, such that the Brauer class corresponding to $X^{(k)}$ is $\alpha^{k}$. This identification of the relative Jacobian of $X^{(k)} / S$ with $J / S$ depends, however, on knowledge of the fact that $X^{(k)}$ was constructed as the moduli space of stable sheaves of rank 1 , degree $k$ on the fibers of $X / S$. If $X^{(k)} \cong X^{\left(k^{\prime}\right)}$, regarding $X^{(k)}$ as the moduli space of line bundles of degree $k^{\prime}$ on the fibers of $X / S$ may 
give rise to a different identification of $J_{X^{(k)}} / S$ with $J / S$. Thus, all we can conclude is that $\alpha^{k^{\prime}}=\varphi\left(\alpha^{k}\right)$ for some automorphism $\varphi$ of $J_{\eta} / \eta$.

The curve $J_{\eta}$ is an elliptic curve over the non-algebraically closed field $\eta$. An automorphism $\varphi$ of $\mathrm{J}_{\eta} / \eta$ could either be fixing the origin of $\mathrm{J}_{\eta}$, or act by translation by a non-zero section, or a combination of both. Consider first the case when the origin is fixed by $\varphi$. We can regard $\varphi$ as an automorphism of the fibration $\mathrm{Ju} / \mathrm{U}$ over some Zariski open set $\mathrm{U}$ in $\mathrm{S}$. Possibly restricting $\mathrm{U}$ further, we can assume that all the fibers of $\mathrm{J} \mathrm{u} / \mathrm{U}$ are smooth, and thus we get automorphisms of these smooth elliptic curves over an algebraically closed field, fixing the origin. Since the fibration is not isotrivial, by [11, IV.4.7] such an automorphism has got to be negation along the fibers of Ju/U. The corresponding action on $\operatorname{Br}\left(\mathrm{J}_{\eta} / \eta\right)$ maps $\alpha^{k}$ to $\alpha^{-k}$. Therefore we conclude that, with regards to automorphisms fixing the origin, the only possibility is for $k^{\prime}= \pm k \bmod n$.

We must rule out now the possibility of the existence of a translation automorphism. Such an automorphism must act as translation by a non-zero section of $\mathrm{J}_{\eta} / \eta$. Such a section must be torsion: otherwise its closure over all of $\mathrm{J} / \mathrm{S}$ would give a divisor which would be linearly independent from either the zero section of $\mathrm{J}$, or from any divisor pulled back from $\mathrm{S}$. Thus we'd have $\mathrm{rkCl} / \mathrm{S}>2$, contradicting the well-known fact (see, for example, 9] that $\mathrm{rkClJ} / \mathrm{S}=\mathrm{rkClX} / \mathrm{S}$, which is 2 .

Assume that $\mathrm{J} / \mathrm{S}$ has a torsion section. Consider a general smooth curve $\mathrm{C}$ in $\mathrm{S}$, and consider the restriction $\mathrm{JC}_{\mathrm{C}} \rightarrow \mathrm{C}$ of $\mathrm{J}$ to $\mathrm{C}$. It is a smooth elliptic fibration of dimension 2 , and the non-trivial torsion section of $\mathrm{J}$ restricts to a non-zero torsion section of $\mathrm{J} \mathrm{C} / \mathrm{C}$. Its fibers are not multiple or reducible, since the original fibration only had isolated such fibers. It is also not isotrivial. But by [8, Proposition 5.3 .4 (ii)] it does not have any torsion sections, which is a contradiction. Thus $\mathrm{J} / \mathrm{S}$ does not have any torsion sections.

The reverse implication is easy and is left to the reader.

2.3. Proposition. If one of the spaces $X^{(k)}$ (which we'll call $X$ without loss of generality) admits two structures of genus one fibration, then $\mathrm{X}$ is the unique smooth projective CalabiYau variety in its birational equivalence class. Otherwise, there may exist at most one more smooth projective Calabi-Yau variety birational to $\mathrm{X}$ admitting a genus one fibration structure.

Proof. This proof is based on ideas from the Mori program. Observe that smooth, projective Calabi-Yau varieties are minimal in the sense of this program. Therefore, all other minimal varieties birational to $X$ differ from $X$ by a finite number of flops.

Since the rank of the Picard group of $X$ is 2 , the nef cone of $X$ is a wedge in $R^{2}$ (see figure above). Faces of this nef cone correspond to extremal contractions of $X$. Each face could correspond to a Mori fiber space (a K3 or genus one fibration, in this case), or to a flopping contraction.

Note that there are precisely two faces of the nef cone, because of the topology of $\mathbf{R}^{2}$. If $X$ admits two structures of genus one fibration, it means that both faces of the Mori cone correspond to Mori fiber spaces, and therefore there are no flopping contractions that can be performed on $X$. Thus $X$ is the unique minimal projective variety in its birational class.

If $X$ admits a unique structure of genus one fibration, this labels one face of the nef cone. The other face of the nef cone could correspond to a K3 fibration on X, or to a flop. 


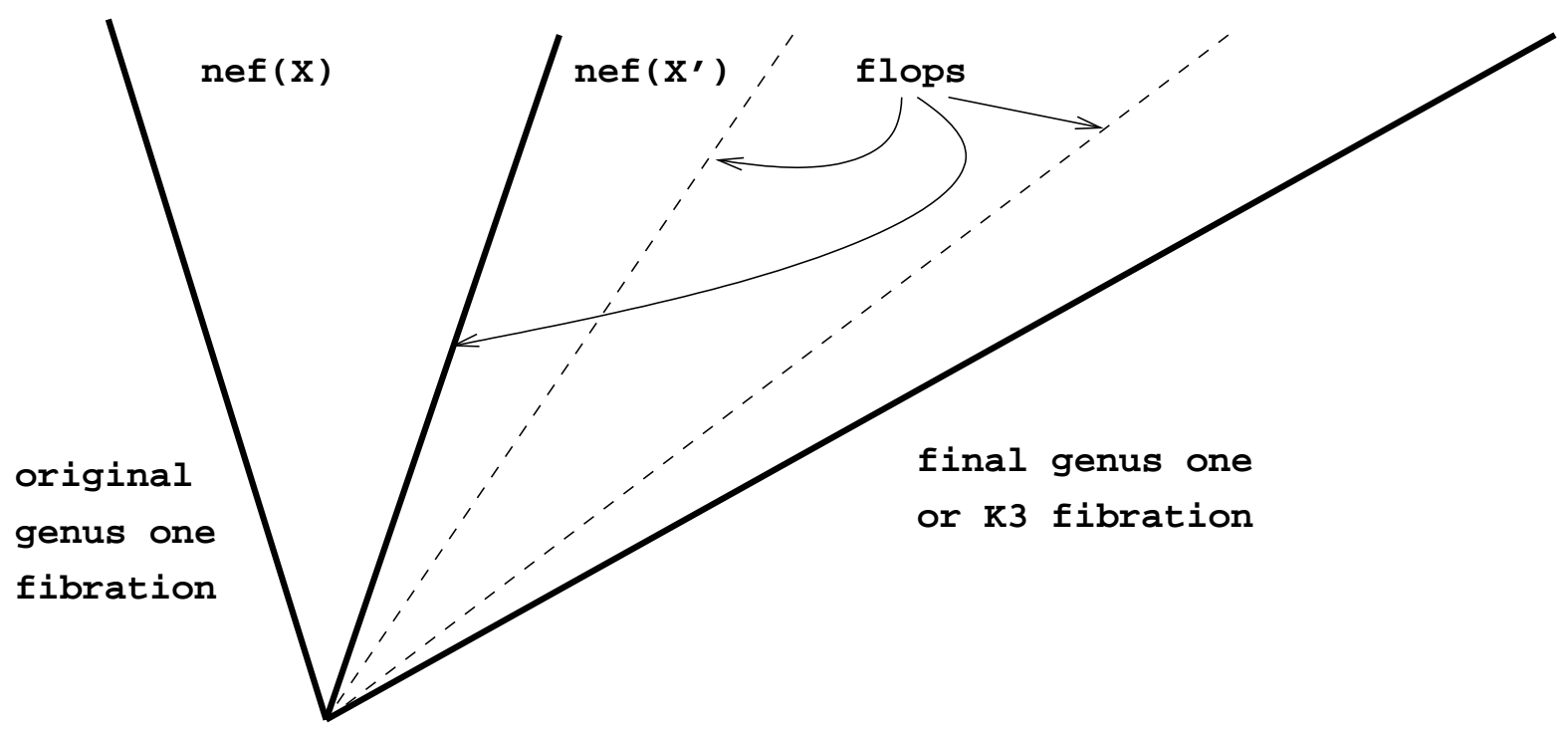

Figure 1: The nef cones of birational models of $X$

In the first case, we are done by an argument similar to the previous case ( $\mathrm{X}$ is unique in its birational class). Otherwise, let $X^{\prime}$ be the result of flopping $X$ along the corresponding side of the nef cone. The nef cone of $X^{\prime}$ is a new wedge in $\mathbf{R}^{2}$, adjacent to the nef cone of $X$.

Now consider the new face of the nef cone of $X^{\prime}$. It could again yield a Mori fiber space. If it does, our analysis of minimal models of $X$ is finished $\left(X\right.$ and $X^{\prime}$ are the only minimal varieties birational to $X$, because the only flopping contraction of $X$ yields $X^{\prime}$, and vice versa). The Mori fiber space structure on $X^{\prime}$ could be that of a genus one fibration, or a $\mathrm{K} 3$ fibration, but in any case this yields at most one more variety birational to $\mathrm{X}$ with a genus one fibration structure, and the process has stopped.

If, on the other hand, the new face of the nef cone of $X^{\prime}$ yields a flopping contraction, then $X^{\prime}$ does not have any genus one fibration structure. Indeed, any such structure must arise from a Mori fiber space-type face of the nef cone, and both faces of nef $(X)$ yield flops, not fiber spaces. By flopping the new ray gives rise to a new space minimal birational model $X^{\prime \prime}$ of $X$, but $X^{\prime}$ itself does not need to be considered for examples of genus one fibrations birational to $X$.

This process can now be continued. At each step we consider the new face of the nef cone of the last space constructed. If this face yields a Mori fiber space, we might have arrived at one more genus one fibration on a space birational to $X$, but the generating process of birational models of $X$ has stopped. Or, if this face yields a flop, we generate a new minimal model of $X$, but the current space does not have any genus one fibration structure. Only the ends of the chain of nef cones can yield minimal models of $X$ with genus one structure. One of them is $\mathrm{X}$ itself, and there can be at most one other such end.

2.4. Theorem. If a space $X$ satisfying the properties required by Theorem 0.1 exists, then 
there exist smooth, projective Calabi-Yau threefolds $X^{\prime}$ and $X^{\prime \prime}$ that are Fourier-Mukai equivalent, but not birational.

Proof. Consider the collection $\mathscr{C}=\left\{X^{(k)}\right\}$ for $k$ coprime to $n, 1 \leq k \leq(n-1) / 2$.

If all the spaces in $\mathscr{C}$ admit a unique structure of genus one fibration (which must be the one arising from regarding them as relative moduli spaces on $\mathrm{X} / \mathrm{S}$ ), then Proposition 2.2 shows that they must all be distinct. By the assumptions made on $\mathrm{n}$, there are at least 3 of them. On the other hand, by Proposition 2.3 they can not all be birational to $\mathrm{X}$, thus the conclusion of the theorem.

If one of the $X^{(\mathrm{k})}$ 's in the collection admits two genus one fibration structures, then it is unique in its birational equivalence class. If any other space $X^{\left(k^{\prime}\right)}$ in $\mathscr{C}$ is non-isomorphic to it, then $X^{(k)}$ and $X^{\left(k^{\prime}\right)}$ is a pair of Fourier-Mukai equivalent, non-birational Calabi-Yau threefolds.

The only case left to consider is if all the spaces in $\mathscr{C}$ are isomorphic to each other, each admitting precisely two structures of genus one fibration. Note that on each space in $\mathscr{C}$, there is a distinguished such structure, arising from their construction as moduli spaces on $X / S$. Since there are at least 3 different allowable values of $k$, we must be able to find $k$ and $k^{\prime}$ such that $X^{(k)} / S$ and $X^{\left(k^{\prime}\right)} / S$ are isomorphic as fibrations, with the induced structure from $\mathrm{X} / \mathrm{S}$. This contradicts Proposition [2.2, and therefore this case is not possible.

\section{An isomorphism of Hodge structures}

In this section we argue first that any two Calabi-Yau threefolds that are Fourier-Mukai equivalent have isomorphic Z[1/2]-Hodge structures. We then argue that if we can regard one of the spaces $X^{\prime}$ and $X^{\prime \prime}$ constructed in the previous section as the moduli space of stable rank 2 vector bundles on the fibers of the other, then they have isomorphic Z-Hodge structures.

3.1. Proposition. Let $\mathrm{X}$ and $\mathrm{Y}$ be Calabi-Yau threefolds, (complex projective manifolds, simply connected and with trivial canonical class) such that $\mathbf{D}(\mathrm{X}) \cong \mathbf{D}(\mathrm{Y})$. Then there exists a Hodge isometry

$$
H^{3}(X, Z[1 / 2])_{\text {free }} \cong H^{3}(Y, Z[1 / 2])_{\text {free }},
$$

where the intersection form on these groups is given by the cup product, followed by evaluation against the fundamental class of the space, and the subscript "free" denotes the torsion-free part of the corresponding group.

If $\mathrm{U}^{\cdot}$ is an element of $\mathrm{D}(\mathrm{X} \times \mathrm{Y})$ that induces an isomorphism $\mathrm{D}(\mathrm{X}) \cong \mathrm{D}(\mathrm{Y})$, denote by $\mathrm{c}_{3}^{\prime}\left(\mathrm{U}^{\cdot}\right)$ the component of $\mathrm{c}_{3}\left(\mathrm{U}^{\cdot}\right)$ that lies in $\mathrm{H}^{3}(\mathrm{X}, \mathbf{Z}) \otimes \mathrm{H}^{3}(\mathrm{Y}, \mathbf{Z})$ under the Künneth decomposition. If $\mathrm{c}_{3}^{\prime}\left(\mathrm{U}^{\cdot}\right)$ is divisible by 2 , the isometry between the $\mathrm{H}^{3}$ groups described above is integral, i.e. it restricts to a Hodge isometry

$$
H^{3}(X, Z)_{\text {free }} \cong H^{3}(Y, Z)_{\text {free }}
$$


3.2. It is worth observing that $\mathrm{H}^{3}$ is the only cohomology group of a Calabi-Yau threefold that carries any Hodge structure data. Thus the above Proposition refers to all the Hodge information of such a space.

There is, however, one more topological information that may not agree between $X$ and $\mathrm{Y}$, despite the above theorem. It is the cubic form on $\mathrm{H}^{2}$, obtained by taking a class in $\mathrm{H}^{2}$, cubing it, and then integrating against the fundamental class.

This is not too big a problem, though. In general, when looking for counterexamples to Torelli, we want examples that are in the same family. In our case, this would have to be analyzed by some other external means, depending on the explicit geometry of the genus one fibration we start with. If we know that $X^{\prime}$ and $X^{\prime \prime}$ are deformation equivalent, then their cubic forms on $\mathrm{H}^{2}$ will be the same.

3.3. Warning: Throughout this section we will use a non-standard notation, by using $\mathrm{H}^{\mathrm{p}, \mathrm{q}}$ for the subgroup $\mathrm{H}^{\mathrm{p}}(\mathrm{X}, \mathbf{C}) \otimes \mathrm{H}^{\mathrm{q}}(\mathrm{Y}, \mathbf{C})$ of $\mathrm{H}^{\mathrm{p}+\mathrm{q}}(\mathrm{X} \times \mathrm{Y}, \mathbf{C})$ (from the Künneth decomposition), instead of the one that comes from the Hodge decomposition.

Proof. Using [17, Theorem 2.2], we can assume that the isomorphism $\mathbf{D}(X) \cong \mathbf{D}(Y)$ is given by a Fourier-Mukai transform $\Phi_{X \rightarrow Y}^{\mathrm{U}^{\cdot}}$ for some $\mathrm{U}^{\cdot} \in \mathrm{D}(\mathrm{X} \times \mathrm{Y})$. By (1.4) we get an isometry

$$
\varphi: H^{*}(X, C) \cong H^{*}(Y, C),
$$

where the two groups are endowed with the generalized Mukai pairing. This isometry must take $\mathrm{H}^{3}(\mathrm{X}, \mathbf{C})$ to $\mathrm{H}^{3}(\mathrm{Y}, \mathbf{C})$ and vice-versa. Indeed, $\mathrm{H}^{3}$ contains all the odd cohomology of a Calabi-Yau threefold, and because the isometry respects the Hochschild grading, it must map $H^{\text {odd }}(X)$ to $H^{\text {odd }}(Y)$. It is easy to see that the restriction of the Mukai pairing from $\mathrm{H}^{*}(\mathbf{X}, \mathbf{C})$ to $\mathrm{H}^{3}(\mathbf{X}, \mathbf{C})$ agrees with the usual Poincaré inner product (up to a constant $-\mathrm{i}$ sign). Thus $\varphi$ is an isometry $\mathrm{H}^{3}(\mathrm{X}, \mathrm{C}) \cong \mathrm{H}^{3}(\mathrm{Y}, \mathrm{C})$.

Observe also that $\varphi$ respects the usual Hodge decomposition of $\mathrm{H}^{3}$ : each one of the terms of the Hodge decomposition is unique in its Hochschild graded piece (1.3). Thus $\varphi$ is a Hodge isometry.

The correspondence $\varphi$ is given by

$$
\varphi(\cdot)=\pi_{\mathrm{Y}, *}\left(\pi_{\mathrm{X}}^{*}(\cdot) \cdot \operatorname{ch}\left(\mathrm{U}^{\cdot}\right) \cdot \sqrt{\operatorname{td}(\mathrm{X} \times \mathrm{Y})}\right) .
$$

Write $u_{i}$ for $c_{i}\left(U^{\cdot}\right)$, and $r$ for $r k\left(U^{*}\right)$.

Observe that the only part that contributes to the $H^{3}(Y, C)$ component of $\varphi(x)$ (for some $\left.x \in \mathrm{H}^{3}(X, C)\right)$ is the $\mathrm{H}^{6,3}$ part of

$$
\pi_{X}^{*}(x) \cdot \operatorname{ch}\left(\mathrm{U}^{\cdot}\right) \cdot \sqrt{\operatorname{td}(\mathrm{X} \times \mathrm{Y})},
$$

and thus, since $\pi_{X}^{*}(x) \in H^{3,0}$, we are only interested in the $H^{3,3}$ component of

$$
\operatorname{ch}\left(\mathrm{U}^{\cdot}\right) \cdot \sqrt{\operatorname{td}(\mathrm{X} \times \mathrm{Y})}
$$

Now

$$
\sqrt{\operatorname{td}(X \times Y)}=1+\frac{1}{24}\left(\pi_{X}^{*} c_{2}(X)+\pi_{Y}^{*} c_{2}(Y)\right)+\text { higher order terms, }
$$


so that the $\mathrm{H}^{3,3}$ component of

$$
\operatorname{ch}\left(\mathrm{U}^{\cdot}\right) \cdot \sqrt{\operatorname{td}(\mathrm{X} \times \mathrm{Y})}
$$

is just $\mathrm{ch}_{3}\left(\mathrm{U}^{\cdot}\right)$. Indeed, $\pi_{\mathrm{X}}^{*} \mathrm{c}_{2}(\mathrm{X}) \in \mathrm{H}^{4,0}$, so it can not give an element of $\mathrm{H}^{3,3}$ by multiplication with anything. We have

$$
\mathrm{ch}_{3}\left(\mathrm{u}^{\cdot}\right)=\frac{1}{6}\left(\mathrm{c}_{1}^{3}\left(\mathrm{u}^{\cdot}\right)-3 \mathrm{c}_{1}\left(\mathrm{U}^{\cdot}\right) \mathrm{c}_{2}\left(\mathrm{U}^{\cdot}\right)+3 \mathrm{c}_{3}\left(\mathrm{U}^{\cdot}\right)\right)
$$

$c_{1}\left(U^{\cdot}\right) \in H^{2}(X \times Y, C)$, and since $H^{1}(X, C)=H^{1}(Y, C)=0$, it must belong to $H^{2,0} \oplus H^{0,2}$. Therefore $c_{1}^{3}\left(U^{*}\right)$ can not have any $H^{3,3}$ component. Similarly for $c_{1}\left(U^{*}\right) c_{2}\left(U^{*}\right)$. Hence we conclude that the only contribution to the map $\mathrm{H}^{3}(X, \mathbf{C}) \rightarrow \mathrm{H}^{3}(\mathrm{Y}, \mathbf{C})$ comes from $\mathrm{c}_{3}\left(\mathrm{U}^{\cdot}\right)$, in the form

$$
\left.\varphi\right|_{\mathrm{H}^{3}(\mathrm{X}, \mathrm{C})}(\cdot)=\frac{1}{2} \pi_{\mathrm{Y}, *}\left(\pi_{\mathrm{X}}^{*}(\cdot) \cdot \mathrm{c}_{3}^{\prime}\left(\mathrm{U}^{\cdot}\right)\right)
$$

and hence the result.

3.4. Proposition. Just as before, let $X$ and $Y$ be Calabi-Yau threefolds with equivalent derived categories. Assume that there exists a codimension 2 subvariety $i: Z \hookrightarrow X \times Y$ which is integral and locally a complete intersection, and an element $\mathrm{U} \in \mathbf{D}_{\text {perf }}(\mathbf{Z})$ such that if one takes $i_{*} \mathrm{U}^{\cdot} \in \mathbf{D}(\mathrm{X} \times \mathrm{Y})$, then $\Phi_{X \rightarrow Y}^{i_{*} \mathrm{U}^{*}}: \mathbf{D}(X) \rightarrow \mathbf{D}(\mathrm{Y})$ is an equivalence of categories. Assume furthermore that $\mathrm{U}$ has rank 2 on Z. Then the isometry of Proposition [3.1 is integral, i.e. it restricts to a Hodge isometry

$$
H^{3}(X, Z)_{\text {free }} \cong H^{3}(Y, Z)_{\text {free }} .
$$

Proof. As seen before, to prove the integrality of the isomorphism we would need to show that $c_{3}\left(i_{*} U^{\cdot}\right)$ is divisible by 2 . We use Grothendieck-Riemann-Roch to compute $c_{3}\left(i_{*} U^{\cdot}\right)$ (we can apply it because $i$ is a locally complete intersection projective morphism). We have

$$
\operatorname{ch}\left(i_{*} \mathrm{U}^{\cdot}\right)=\mathfrak{i}_{*}\left(\operatorname{ch}\left(\mathrm{U}^{\cdot}\right) \cdot \operatorname{td}(-\mathscr{N})\right),
$$

where $\mathscr{N}$ is the normal sheaf of $\mathrm{Z}$ in $\mathrm{X} \times \mathrm{Y}$, and the negation is taken in the Grothendieck group (see [10, Exposé 0]).

The left hand side of the above equality is

$$
r+c_{1}+\frac{1}{2}\left(c_{1}^{2}-c_{2}\right)+\frac{1}{6}\left(c_{1}^{3}-3 c_{1} c_{2}+3 c_{3}\right)+\ldots,
$$

where $c_{i}=c_{i}\left(i_{*} U^{*}\right)$. Obviously, $r=c_{1}=0$ because $Z$ has complex codimension 2 (also from the right hand side of the equality), so the real dimension 6 part of the left hand side consists of just

$$
\frac{1}{2} c_{3}\left(i_{*} u^{\cdot}\right)
$$

On the other hand, the real dimension 6 part of the right hand side comes from the dimension 2 part of

$$
\operatorname{ch}\left(\mathrm{U}^{\cdot}\right) \cdot \operatorname{td}(-\mathscr{N})
$$


which consists of

$$
\mathrm{c}_{1}\left(\mathrm{U}^{\cdot}\right)-\frac{1}{2} \mathrm{rk}\left(\mathrm{U}^{*}\right) \mathrm{c}_{1}(\mathscr{N})
$$

We conclude that

$$
\mathrm{c}_{3}\left(i_{*} \mathrm{U}^{\cdot}\right)=2 i_{*} \mathrm{c}_{1}\left(\mathrm{U}^{\cdot}\right)-\operatorname{rk}\left(\mathrm{U}^{\cdot}\right) i_{*} \mathrm{c}_{1}(\mathscr{N}) .
$$

The first term is obviously divisible by 2 , and the second one is divisible by 2 because we have assumed $r k\left(\mathrm{U}^{*}\right)=2$. The result follows.

3.5. We conjecture that the arguments of the previous section can be repeated to get two spaces $X^{\prime}$ and $X^{\prime \prime}$ which are Fourier-Mukai equivalent, non-biratonal, and having the following property: one can be regarded as a component of the moduli space of stable torsion free sheaves of rank 2 , degree $k$ on the other one, for some $k$.

If this is the case, then probably Proposition 3.4 can be applied to these spaces (the universal sheaf $U$ would be supported on $X^{\prime} \times{ }_{S} X^{\prime \prime}$ which is codimension 2 in $X^{\prime} \times X^{\prime \prime}$, and we in fact expect $U$ to be a rank 2 vector bundle on $\left.X^{\prime} \times_{S} X^{\prime \prime}\right)$. Then $X^{\prime}$ and $X^{\prime \prime}$ would have isomorphic Z-Hodge structures, thus giving a complete example of two non-birational Calabi-Yau varieties with isomorphic Hodge structures.

3.6. The reason we expect to be able to regard $X^{\prime \prime}$ as a moduli space of rank 2 (instead of rank 1) vector bundles on the fibers of $X^{\prime}$ is the following. Given an elliptic curve $E$, and coprime integers $r$ and $d$ with $r>0$, then the moduli space of stable vector bundles on $E$ of rank $r$, degree $d$ is again isomorphic to $E$ (but the choice of isomorphism depends on the choice of origin of $E$ ). Thus starting with $X$, and considering relative moduli spaces of stable sheaves of rank 2 , degree $k$ on the fibers of $X / S$ for various $k$, we get genus one fibrations. The relative Jacobian of these fibrations is isomorphic to $\mathrm{J} / \mathrm{S}$, as these fibrations have the same fibers as $X / S$. We believe it is highly unlikely that these new fibrations would be represented in $\operatorname{Br}(\mathrm{J} / \mathrm{S})$ by anything other than a power of $\alpha$-there does not appear to be any good way to create from nothing new classes in the Brauer group.

If the above reasoning is correct, then the spaces we are considering are again the $X^{(k)}$ 's we have considered in Section 2. but now regarded as moduli spaces of rank 2 vector bundles. This does not yet say that any one of them can be regarded as a moduli space of rank 2 vector bundles on any other one of them. But we expect that there exists a simple formula of the form

$$
X^{\left(f\left(2, k, k^{\prime}, n\right)\right)}=\mathscr{M}_{X^{(k)}}\left(0,2, k^{\prime}\right)
$$

where $f$ is a simple polynomial in $k, k^{\prime}$ and $n$, and $\mathscr{M}_{\chi^{(k)}}\left(0,2, k^{\prime}\right)$ represents the moduli space of rank 2, degree $k^{\prime}$ stable sheaves on the fibers of $X^{(k)}$. Then, for an appropriate choice of $n$, one would be able to argue that every $X^{(k)}$ is a moduli space of stable sheaves of rank 2, degree $l$ on the fibers of any other such space.

\section{References}

[1] Bondal, A., Orlov, D., Semiorthogonal decompositions for algebraic varieties, preprint, alg-geom/9506012 
[2] Bridgeland, T., Fourier-Mukai transforms for elliptic surfaces, J. reine angew. math. 498 (1998) 115-133 (also preprint, alg-geom/9705002)

[3] Bridgeland, T., Flops and derived categories, Invent. Math. 147 (2002), no. 3, 613-632

[4] Bridgeland, T., Maciocia, A., Complex surfaces with equivalent derived categories, Math. Z. 236 (2001), no. 4, 677-697

[5] Căldăraru, A., Derived Categories of Twisted Sheaves on Calabi-Yau Manifolds, Ph.D. thesis, Cornell University (2000), also available at http://www.math.wisc.edu/ ${ }^{2}$ andreic/publications/ThesisSingleSpaced.pdf

[6] Căldăraru, A., Derived categories of twisted sheaves on elliptic threefolds, J. Reine Angew. Math. 544 (2002), 161-179

[7] Căldăraru, A., The Mukai pairing. II. The Hochschild-Kostant-Rosenberg isomorphism, Adv. Math. 194 (2005), no. 1, 34-66

[8] Cossec, F., Dolgachev, I., Enriques Surfaces I, Birkhäuser, Boston, (1989)

[9] Dolgachev, I., Gross, M., Elliptic three-folds I: Ogg-Shafarevich theory, J. Alg. Geom. 3 (1994), 39-80 (also preprint, alg-geom/9210009)

[10] Grothendieck, A., Berthelot, P., Illusie, L., et al, Séminaire de Géométrie Algébrique 6: Théorie des Intersections et Théorème de Riemann-Roch, Lecture Notes in Math. 225, Springer-Verlag, Heidelberg (1971)

[11] Hartshorne, R., Algebraic Geometry, Graduate Texts in Mathematics Vol. 52, Springer-Verlag (1977)

[12] Kapustin, A., Rozansky, L., On the relation between open and closed topological strings, Comm. Math. Phys. 252 (2004)

[13] Katz, S., Sharpe, E., D-branes, open string vertex operators, and Ext groups, Adv. Theor. Math. Phys. 6 (2002), no. 6, 979-1030 (2003)

[14] Kontsevich, M., Homological algebra of mirror symmetry, Proceedings of the 1994 International Congress of Mathematicians I, Birkäuser, Zürich, 1995, p. 120 (also preprint, alg-geom/9411018)

[15] Mukai, S., Duality between $\mathrm{D}(\mathrm{X})$ and $\mathrm{D}(\hat{\mathrm{X}})$ with its application to Picard sheaves, Nagoya Math. J., Vol. 81 (1981), 153-175

[16] Mukai, S., On the moduli space of bundles on K3 surfaces, I., in Vector Bundles on Algebraic Varieties, Oxford University Press (1987), 341-413

[17] Orlov, D., Equivalences of derived categories and K3 surfaces, J. Math. Sci. (New York) 84 (1997), 1361-1381 (also preprint, math.AG/9606006) 
[18] Simpson, C. T., Moduli of representations of the fundamental group of a smooth projective variety, I, Publ. Math. IHES, 79 (1994), 47-129

[19] Szendrői, B., Calabi-Yau threefolds with a curve of singularities and counterexamples to the Torelli problem, Internat. J. Math. 11 (2000), no. 3, 449-459, also preprint, alg-geom/9901078

[20] Toda, Y., Fourier-Mukai transforms and canonical divisors, preprint, math.AG/0312015 